\title{
A Mathematical Model on Dispersion of Air Pollutants
}

\author{
V. S. Verma ${ }^{1}$, Uma Srivastava ${ }^{2}$, P. S. Bhandari ${ }^{3}$ \\ ${ }^{1}$ Department of Mathematics and Statistics, D.D.U. Gorakhpur University, Gorakhpur-273009, U.P., India \\ ${ }^{3}$ Department of Mathematics, Birendra Multiple Campus, Tribhuvan University, Nepal
}

\begin{abstract}
In this paper, an analytical approach to the problem of dispersion of air pollutants emitted from a point source with variable wind velocity and constant removal rate is proposed to study in steady state condition where eddy diffusivity coefficients are taken as constants. The methods of separation of variables and Fourier transform have been used for the solution of the problem.
\end{abstract}

Keywords: Air pollutant, concentration profile, variable wind velocity, constant removal rate

\section{Introduction}

Air pollution is a growing concern of the most countries in the world whether it is developed or developing. The increasing number of automobiles, growing number of industrial plants and less use of recycling of industrial waste materials are the basic causes of air pollution. Emission of different types of harmful gases and particles are the basic root cause of environmental pollution. Due to the environmental pollution human beings, animals and plants are facing different types of problem.

To protect ourselves from contaminants released to the atmosphere, it is better to understand the physical phenomenon involved in the atmospheric pollutant dispersion. In order to reduce the pollutants emission in the atmosphere it is necessarily to monitor air quality constantly. Therefore, precise modeling of pollutants concentration near earth surroundings is significantly important.

Atmospheric dispersion of pollutants has attracted researchers in many ways. Some have focused in the environmental impact and health hazards, while others have worked on various modelling aspect such as meteorological conditions, dispersion mechanism, removal mechanisms, topographical features, etc. Invariably, mathematical modeling has been the case of the most of these studies. Several models have been discussed in the past to deal with air pollution dispersion under different atmospheric conditions.

In particular, Sirakov and Djolov [2] had made an attempt to model the dispersion of pollutants from a continuous source in absence of the wind while Demuth et al. [1] have presented an analytical model for calm wind situations when there is a finite mixing height. Sharan et al. [4] have given a mathematical model for the dispersion of air pollutants in low wind conditions by assuming constant eddy diffusivity coefficients in the advection diffusion equation.

Sharan et al. [5] have given an overview of mathematical modelling framework of atmospheric dispersion. Agarwal et al. [3] have presented an analytical model to the problem of dispersion of an air pollutant with variable wind velocity. Srivastava et al. [10] have given a three dimensional atmospheric diffusion model with variable removal rate and variable wind velocity using power law profile. Kumar et al. [7] have presented an analytical model for the dispersion of pollutant released from a continuous source in the atmospheric boundary layer describing the crosswindintegrated concentration. They have claimed that the model can also be used for the concentration distribution of a pollutant released from a line source perpendicular to the direction of the mean wind and to study the turbulent dispersion from a steady two dimensional horizontal source in a wide open channel for a generalized profile of wind flow and eddy diffusivity.

Verma et al. [11] have given an analytical approach to the problem of dispersion of an air pollutant with variable wind velocity and variable eddy diffusivity. They have taken wind velocity in the form of wave function. Sharan [6] have proposed a steady-state mathematical model for the dispersion of air pollutants in low winds by taking into account the diffusion in the three coordinate directions and advection along the mean wind. Verma [12] have proposed an analytical approach to the problem of dispersion of an air pollutant with constant wind velocity and constant removal rate. In his paper, it is found that the concentration profile of air pollutant becomes high near the ground but as the distance from the ground increases, the concentration profile decreases regularly. Khaled et al. [9] have presented a mathematical model of the atmospheric diffusion equation and have analysed the advection diffusion equation in two directions to obtain the crosswind integrated concentration. The effect of various parameters on the associated results is given by many researchers, but no attempts have been made with variable wind velocity and constant removal rate in the steady state condition. Therefore in this paper, we have made an attempt to the solution of dispersion equation for the concentration of air pollutions emitted from a point source with variable wind velocity and constant removal rate in the steady state condition where eddy diffusivity coefficients are taken as constant.

\section{Requirement of Dispersion Model}

There are several competing requirements in the design of an air pollution model. A model captures the essential physics of dispersion process and provides reasonable and

\section{Volume 4 Issue 12, December 2015}




\section{International Journal of Science and Research (IJSR) \\ ISSN (Online): 2319-7064}

Index Copernicus Value (2013): 6.14 | Impact Factor (2014): 5.611

reputable estimates of downwind concentrations. This generally requires detailed knowledge of source characteristics, terrain and meteorology, but it is also desirable to keep these input requirements to a minimum and simplicity is an important asset in any model. All models should have a fully documented account of the equation algorithms used and their conversion into valid software. In choosing an air dispersion model, several levels of model are available, with progressively increasing levels of mathematical sophistication, input data requirements and user expertise required. At the low end of the scale are the gross screening models, which require only a hand-held calculator, monograph, or spreadsheet. They may treat only one source at a time and provide some sort of worst-case prediction based on relatively primitive meteorological information. It is often wise to apply such a model prior to using the more advanced models, where the flow of information is more difficult to follow.

Specialized models are often used for predicting dispersion of special hazardous materials, such as military models used in chemical/biological defense. Heavy gas dispersion models are used by chemical process industries to model the behaviour of rough or accidental releases of dense gases or vapours.

Although the input data requirements and level of sophistication increase with the more advanced models, a more complex model does not necessarily lead to predictions that are more accurate. As the number of input variables goes up in the advanced models, the room for input data error increases. In addition, the level of user understanding must increase to make proper use of the model (Macdonald [8]).

\section{Mathematical Formulation}

The dispersion of an air pollutant in the atmosphere under steady state condition is described by the following partial differential equation

$$
U(x) \frac{\partial C}{\partial x}=K_{y} \frac{\partial^{2} C}{\partial y^{2}}+K_{\mathrm{z}} \frac{\partial^{2} C}{\partial z^{2}}-\alpha C
$$

where $\mathrm{x}, \mathrm{y}, \mathrm{z}$ are the Cartesian co-ordinates, $\mathrm{U}(\mathrm{x})$ is the variable wind velocity which varies with the downwind distance and here it is assumed in the form of a wave function given by $\mathrm{U}(\mathrm{x})=\mathrm{U}_{0}\left[1+\varepsilon \cos \left(\frac{2 \pi x}{\mathrm{Z}}\right)\right]$, where $\mathrm{U}_{0}$ is mean wind velocity, $\lambda$ is the wave length, $\mathrm{C}$ is the concentration of the air pollutant, $\mathrm{K}_{\mathrm{y}}$ and $\mathrm{K}_{\mathrm{z}}$ are the eddy diffusivities in $y$ - and $z$ - directions respectively which are assumed to be constants and $\alpha$ is the removal rate of the air pollutant due to some natural mechanism like chemical reaction, which is also taken to be constant. Typically $\mathrm{K}_{\mathrm{y}}>\mathrm{K}_{\mathrm{z}}$ in the atmosphere.

The boundary conditions for the equation (3.1) are taken as follows:

$$
\begin{array}{lc}
\mathrm{C}(\mathrm{x}, \mathrm{y}, \mathrm{z})=\frac{Q \partial(\mathrm{y}) Q\left(\mathrm{z}-h_{\mathrm{s}}\right)}{W(\mathrm{x})}, & \mathrm{x}=0,0 \leq h_{\mathrm{s}} \leq \mathrm{H} \\
\mathrm{C}(\mathrm{x}, \mathrm{y}, \mathrm{z})=0, & \mathrm{y} \rightarrow \pm \infty \\
\mathrm{C}(\mathrm{x}, \mathrm{y}, \mathrm{z})=0, & \mathrm{z}=0 \\
K_{\mathrm{z}} \frac{\partial C}{\partial z}=v_{\mathrm{d}} \mathrm{C}, & \mathrm{z}=\mathrm{H}
\end{array}
$$

where $\delta$ is the Dirac delta-function, $\mathrm{Q}$ is the emission strength of an elevated point source, $h_{s}$ is the stack height and $v_{\mathrm{d}}$ is the deposition velocity of the air pollutant. Condition (3.2) states that the air pollutant is released from the elevated point source of strength Q. Condition (3.3) states that the concentration of the air pollutant is zero for $y$ $\rightarrow \pm \infty$. Condition (3.4) states that the concentration of the air pollutant is zero at the ground surface and condition (3.5) states that there is some diffusion flux at the vertical height $\mathrm{H}$ from the ground surface.

\section{Method of Solution}

Before solution, the partial differential equation (3.1) describing the dispersion of air pollutant and the boundary conditions are made non-dimensional by introducing the following non- dimensional quantities:

$$
\begin{aligned}
& \mathrm{X}^{*}=\frac{K_{z_{0}} X^{X}}{\mathrm{U}_{0} H^{2}}, y *=\frac{y}{H}, \mathrm{Z}^{*}=\frac{z}{H}, \mathrm{U}^{*}=\frac{\mathrm{U}(x)}{\mathrm{U}_{0}},
\end{aligned}
$$

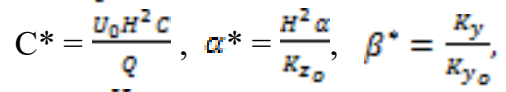

$$
\begin{aligned}
& y^{*}=\frac{K_{z}}{K_{z_{\rho}}}, \quad \delta\left(y^{*}\right)=H \delta(y), \quad N^{*}=\frac{H v_{d}}{K_{z}}
\end{aligned}
$$

where $\mathrm{U}_{0}$ is the reference wind velocity and $K_{z_{\rho}}$ is the reference diffusivity.

On dropping asterisk (*), the equation (3.1) and the boundary conditions (3.2) - (3.5) may be put in non dimensional form as given below

$$
\begin{array}{ll}
{\left[1+\epsilon \cos \left(\frac{2 \pi x}{2}\right)\right] \frac{\partial C}{\partial x}=\beta \frac{\partial^{2} C}{\partial y^{2}}+\gamma \frac{\partial^{2} C}{\partial z^{2}}-\alpha C} \\
C=\frac{g(y) a\left(z-h_{2}\right)}{\left[1+E \cos \left(\frac{2 \pi x}{2}\right)\right],}, & x=0 \\
C=0, & \mathrm{y} \rightarrow \pm \infty \\
C=0, & \mathrm{z}=0 \\
\frac{\partial C}{\partial z}=N C, & z=1
\end{array}
$$

Now, we solve equation (4.1) with boundary conditions (4.2) - (4.5) by applying Fourier transform technique.

Therefore, taking Fourier transform of (4.1) with respect to $\mathrm{y}$, we get

$$
\left[1+\varepsilon \cos \left(\frac{2 \pi x}{2}\right)\right] \frac{\partial C}{\partial z}=-\left(p^{2} \beta+\alpha\right) \bar{C}+\gamma \frac{\partial^{2} C}{\partial z^{2}}
$$

where $\bar{C} \equiv \bar{C}(x, p, z)$ is the Fourier transform of $\mathrm{C} \equiv \mathrm{C}(\mathrm{x}, \mathrm{y}, \mathrm{z})$ with respect to $\mathrm{y}$ and $\mathrm{p}$ is the corresponding Fourier transform parameter.

Taking Fourier transforms of $(4.2)-(4.5)$, the boundary conditions become:

$$
\begin{aligned}
& \bar{C}=\frac{d\left(z-h_{s}\right)}{\left[1+E \cos \left(\frac{2 \pi x}{2}\right)\right]}, \quad . \quad x=0 \\
& \bar{C}=0 \quad y \quad y \rightarrow \pm \infty \\
& \bar{C}=0 \quad \therefore \quad z=0 \\
& \frac{\partial C}{\partial z}=N \bar{C} \quad v \quad z=1
\end{aligned}
$$

Again, to solve equation (4.6), we shall use the method of separation of variables and thus we assume the trial solution of equation (4.6) in the following form:

$$
\bar{C}=\mathrm{X}(\mathrm{x}) \mathrm{Z}(\mathrm{z})
$$

where $X(x)$ is a function of $x$ only and $Z(z)$ is a function of $z$ only.

Using (4.11) in equation (4.6) and simplifying, we get

$$
\frac{\mathrm{z}^{\mathrm{v}}}{\mathrm{z}}=\left[\frac{\left[1+\varepsilon \cos \left(\frac{2 \pi x}{\lambda}\right)\right.}{\gamma}\right] \frac{x}{x}+\left(\frac{p^{2} \beta+\alpha}{Y}\right)=-\chi^{2}
$$




\section{International Journal of Science and Research (IJSR) \\ ISSN (Online): 2319-7064 \\ Index Copernicus Value (2013): 6.14 | Impact Factor (2014): 5.611}

where $\lambda^{2}$ is a separation constant.

Taking the first and third ratios, we have

$$
Z^{\prime \prime}+\lambda^{2} Z=0
$$

Taking the second and third ratios, we have

$$
\left[\frac{\left[1+\varepsilon \cos \left(\frac{4 \pi x}{\lambda}\right)\right.}{Y}\right] \frac{x}{x}+\left(\frac{p^{2} \beta+\alpha}{Y}\right)+\lambda^{2}=0
$$

where $\lambda^{2}$ is a separation constant.

Solutions of equations (4.13) and (4.14) are respectively given by

$$
\begin{gathered}
\mathrm{Z}(\mathrm{z})=\mathrm{A} \cos (\lambda \mathrm{z})+\mathrm{B} \sin (\lambda \mathrm{z}) \\
\mathrm{X}(\mathrm{x})=\mathrm{M} \exp \left\{-\left(\left\{\mathrm{p}^{2} \beta+\alpha+\lambda^{2} \gamma\right) g(x)\right\}\right.
\end{gathered}
$$

where $\mathrm{A}, \mathrm{B}$ and $\mathrm{M}$ are arbitrary constants and

$$
g(x)=\frac{\mathrm{z}}{\pi \sqrt{1-\varepsilon^{2}}} \tan ^{-1}\left[\tan \left(\frac{\pi x}{\mathrm{z}} \sqrt{\frac{1-\varepsilon}{1+\varepsilon}}\right)\right]
$$

Therefore, putting the values of $\mathrm{Z}(\mathrm{z})$ from (4.15) and $\mathrm{X}(\mathrm{x})$ from (4.16) in (4.11), we get

$$
\bar{C}=\{\mathrm{A} \cos (\lambda \mathrm{z})+\mathrm{B} \sin (\lambda \mathrm{z})\} \exp \left[-\left\{p^{2} \beta+\alpha+\lambda^{2} \gamma\right\} g(x)\right]
$$

where $M$ taken to be 1 , without any loss of generality.

Now, using the boundary conditions $\bar{C}=0, z=0$ and $\frac{\partial C}{\partial z}=N \bar{C}_{x} z=1_{x}$ we get

$$
\tan \left(\lambda_{\mathrm{n}}\right)=\frac{\lambda_{n}}{N^{x}}
$$

where $\mathrm{n}=1,2, \ldots \ldots \ldots \ldots .\left(\frac{\mathrm{n}-1}{2}\right) \pi_{x}$ which is an eigen value equation.

Again, using the boundary condition

$\bar{C}=\frac{g_{\left(z-h_{2}\right]}}{\left[1+E \cos \left(\frac{2 \pi x}{\mathbb{M}}\right)\right]}, \quad x=0$ and as well as applying

$\int_{0}^{1} \delta\left(z-h_{g}\right) f_{n}(z) d z=f_{n}\left(h_{g}\right)$ and

$\int_{0}^{1} z^{p} f_{m}(z) f_{n}(z) d z=0, m \neq n_{x}$

the solution is given by

$$
\begin{gathered}
\bar{C}=\left(\frac{1}{1+\varepsilon}\right) \exp \left[-\left\{p^{2} \beta+\alpha+\lambda_{n}^{2} \gamma\right\} g(x)\right] \\
\sum_{n=1}^{\infty}\left\{\frac{\sin \left(\lambda_{n} z\right) \sin \left(\chi_{n} h_{g}\right)}{p_{n}}\right\}
\end{gathered}
$$

where $\mathrm{P}_{\mathrm{n}}=\int_{0}^{1} \sin ^{2}\left(\eta_{\mathrm{n}} z\right) d z$.

Finally, taking inverse Fourier transform of (4.19), we get

$$
\begin{aligned}
& \quad \mathrm{C}=\left\{\frac{0.28209}{1+\varepsilon}\right\} \frac{1}{\sqrt{\beta g(x)}} \exp \left[-\left\{\alpha g(x)+\frac{y^{2}}{4 \beta g(x)}\right\}\right] \\
& \sum_{n=1}^{\infty}\left\{\frac{\sin \left(\alpha_{m} z\right) \sin \left(m_{n} h_{2}\right)}{P_{n}}\right\} \exp \left\{-\gamma g(x) \lambda_{n}^{2}\right\} \quad \text { where } \mathrm{P}_{\mathrm{n}=} \int_{0}^{1} \sin ^{2}\left(\eta_{n} z\right) d z \quad \text { and } \\
& g(x)=\frac{\mathrm{z}}{\pi \sqrt{1-\varepsilon^{2}}} \tan ^{-1}\left[\tan \left(\frac{\pi x}{\mathrm{z}} \sqrt{\left.\frac{1-\varepsilon}{1+\varepsilon}\right)}\right]\right.
\end{aligned}
$$

\section{Results and Discussion}

To find the effect of various parameters on dispersion of air pollutant, the concentration profile in non-dimensional form is calculated by equation (4.20). The parametric values used in the analysis are taken as follows:

$\alpha=2, \quad \beta=10, \quad \gamma=1, \quad h_{g}=0.2, H=1$,

$\varepsilon=0.005, \lambda=1$
In figure 1 , the concentration profile is plotted against the downwind distance $(0 \leq x \leq 1)$ for different values of vertical distances $(z=0.2,0.4,0.6)$ and the value of $y$ is taken to be zero. It is seen that the concentration profile decreases continuously with increasing downwind distance and it becomes negligible at $\mathrm{x}=1$. It is also observed that the concentration profile decreases with increasing vertical distances $(\mathrm{z}=0.2, \mathrm{z}=0.4, \mathrm{z}=0.6)$ and the decreases in concentration with vertical distance being higher at lower values of downwind distance and at higher values of downwind distance, there is negligible change in the concentration of air pollution with increasing vertical distance.

In figure 2 , the concentration profile is plotted against the downwind distance $(0 \leq x \leq 1)$ for different values of vertical distances $(\mathrm{z}=0.2,0.4,0.6)$ and the value of $\mathrm{y}$ is taken to be one. Here also, it is seen that the concentration profile decreases continuously with increasing downwind distance and it becomes negligible at $x=1$. It is also observed that the concentration profile decreases with increasing vertical distances $(\mathrm{z}=0.2, \mathrm{z}=0.4, \mathrm{z}=0.6)$ and the decreases in concentration with vertical distance being higher at lower values of downwind distance and at higher values of downwind distance, there is negligible change in the concentration of air pollution with increasing vertical distance.

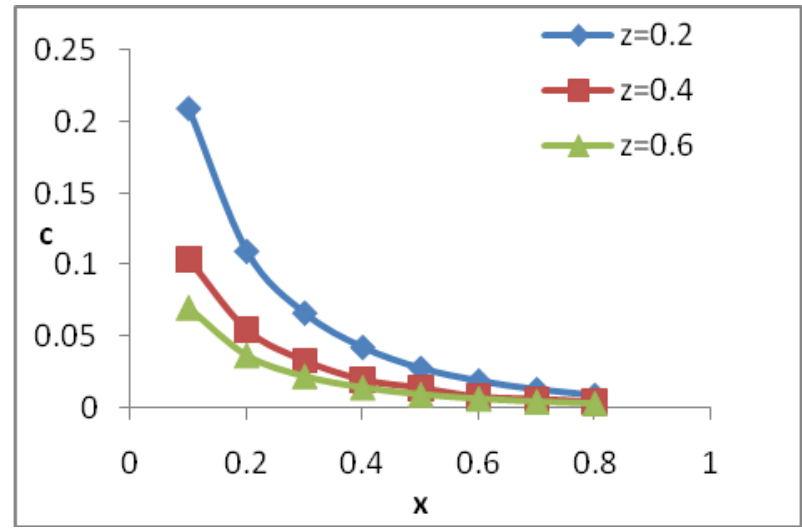

Figure 1: variation of concentration profile with downwind distance $\mathrm{x}$ for different values of vertical distance $\mathrm{z}$ when crosswind distance $\mathrm{y}=0$

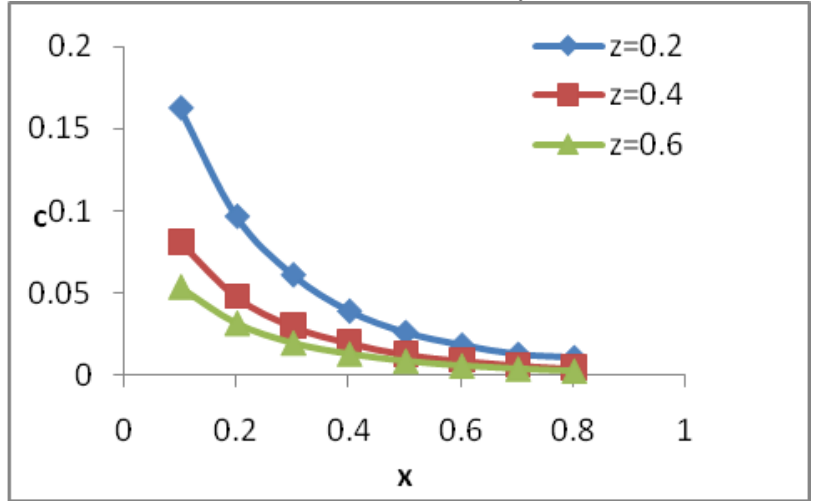

Figure 2: variation of concentration profile with downwind distance $\mathrm{x}$ for different values of vertical distance $\mathrm{z}$ when crosswind distance $\mathrm{y}=1$

\section{Conclusion}




\section{International Journal of Science and Research (IJSR) \\ ISSN (Online): 2319-7064}

Index Copernicus Value (2013): 6.14 | Impact Factor (2014): 5.611

In this model, it is found that the concentration profile of air pollutant decreases continuously with increasing downwind distance and it becomes negligible at $\mathrm{x}=1$. It is also seen that the concentration profile decreases with increasing vertical distances and the decrease in concentration with vertical distance being higher at lower values of downwind distance and at higher values of downwind distance, there is negligible change in the concentration of air pollution with increasing vertical distance.

\section{References}

[1] Cl.Demuth,A.Berger,Y.Jacquart,Ch.Lgros, "Analytical modeling of pollutant dispersion during calm wind situations," In proc. $13^{\text {th }}$ Int. colloquim,Paris,France, 1978.

[2] D.E. Sirakov and G.D.Djolov, "Atmospheric diffusion of admixtures in calm conditions," Geophysique 32,pp.891-892,1979.

[3] M. Agarwal, V. S. Verma and S. Srivastava, "An analytical approach to the problem of dispersion of an air pollutant with variable wind velocity", J. Nat. Acad. Math., 22 pp.51-62, 2008.

[4] M. Sharan and A. K. Yadav, "A mathematical model for the dispersion of air pollutant in low wind conditions", Atmospheric Environment, 30, pp.1209-1220, 1996.

[5] M. Sharan, M. Modani and A. K. Yadav, "Atmospheric dispersion: An overview of Mathematical Modeling Framework", Proc Indian Natn Sci Acad, 69, A, 6, pp.725-744, 2003.

[6] M.Sharan, M.P Singh, and A.K.Yadav, "Mathematcal model for atmospheric dispersion in low winds with eddy diffusivities as linear functions of downwind distance", atmospheric environment 29:16, 1137-1145, 1995.

[7] P.Kumar and M. Sharan, "An analytical model for dispersion of pollutants from a continuous source in the atmospheric boundary layer", Proc. R. Soc. A, pp.383406, 2010.

[8] R. Macdonald,"Theory and objectives of air dispersion modeling", Wind Engineering, MME 47A, pp. 1-11, 2003.

[9] S.M.Essa Khaled, A.N.Mina and H. Mamdouh, "Analytical solution of Diffusion in two dimensions using two forms of eddy difusivites", Rom. Journ. Phys.56, pp.1228-1240, 2011.

[10] S. Srivastava, M. Agarwal and V.S. Verma, "A threedimentional atmospheric diffusion model with variable removal rate and variable wind velocity", J.Nat. Acad. Math. Spl. , pp.189-197, 2009.

[11] V.S.Verma, S. Srivastava and M. Agarwal , "An analytical approach to the problem of Dispersion of an air pollutant with variable wind velocity and variable eddy diffisivity," South East asian J. math. And Math. Sc.,vol.9 (2), pp. 43-48,2011.

[12] V.S.Verma, "An analytical approach to the problem of Dispersion of an air pollutant with constant wind velocity and constant removal rate", Journal of international academy of physical sciences, 15 , pp.4350, 2011.

Volume 4 Issue 12, December 2015 